\title{
An energy conservation indexing method for secure XML data broadcast in mobile wireless networks
}

\begin{abstract}
Secure broadcasting of XML data is becoming an essential requirement for many applications in mobile wireless networks. Several indexing methods have been proposed to reduce the tuning time in processing the XML queries over the wireless XML stream. Tuning time is the sum of period of times which a mobile client stays in active mode in order to retrieve the required data over the wireless stream. Therefore, it is frequently used to estimate the energy consumption of a mobile client. The problem of existing indexing methods is that they cannot directly be applied to an encrypted XML stream since mobile clients can only access the authorized parts of the XML data in an encrypted XML stream. In this paper, we define a unit structure of an XML stream called SecNode which guarantees confidentiality of the XML data in the wireless stream. We also define two indexes called Min (NCS) and Min (NIS) for the SecNode structure based on the set of access authorizations specified in the original XML document in order to efficiently process the XML queries over the encrypted XML stream. Experimental results show that the use of the SecNode structure for secure XML data broadcast improves the performance of XML query processing in terms of tuning time and therefore reduces the power consumption at mobile clients.
\end{abstract}

Keyword: Access control; Data confidentiality; Indexing; Mobile wireless broadcast; XML query processing; XML stream 